Historic, Archive Document

Do not assume content reflects current scientific knowledge, policies, or practices. 



\section{WHOLESALE TRADE LIST SPRING OF 1929}

\section{GILIETT'S \\ FERN AND FIOWER FARM}

KENNETH E. GILLETT, Proprietor

SOUTHWICK, MASSACHUSETTS

Orders accepted under usual trade terms and conditions, boxing at cost.

All of the stock is of my own growing and is now in my nursery ready for shipment.

This list supersedes all previous catalogs and quotations.

Thirty of one variety will be sold at the hundred rate; five at the ten rate; where less than five of one variety are ordered add $25 \%$ to the ten rate.

\section{HARDY NATIVE FERNS}

Adiantum pedatum (Maidenhair Fern)

Per 10 Per 100

Aspidium acrostichoides (Christmas Fer

$\$ 1.00 \quad \$ 8.00$

Aspidium aculeatum Braunii (Prickly Shield Ferm) $\quad 2.30 \quad 20.00$

$\begin{array}{lll}\text { Aspidium cristatum (Crested Wood Fern) } & 1.00 & 8.00\end{array}$

Aspidium cristatum Clintonianum

Aspidium Goldieanum (Goldie's Wood Fern)

Aspidium marginale (Evergreen Wood Fern)

$\begin{array}{rr}1.00 & 8.00 \\ 3.00 & 25.00\end{array}$

$1.00 \quad 8.00$

Aspidium noveboracense (New York Shield Fern) $\quad 1.00 \quad 8.00$

Aspidium spinulosum (Spinulose Wood Fern) $\quad 1.00 \quad 8.00$

Aspidium spinulosum dilatatum

8.00
10.00

$\begin{array}{rr}1.35 & 10.00 \\ 1.00 & 8.00\end{array}$

Asplenium angustifolium (Narrow-leaved Spleenwort) $1.60 \quad 14.00$

Asplenium ebeneum (Ebony Spleenwort) $\quad 1.00 \quad 8.00$

Asplenium Filix-foemina (Lady Fern)

Asplenium thelypteroides (Silvery Spleenwort)

Asplenium trichomanes (Maidenhair Spleenwort)

$1.00 \quad 8.00$

$1.00 \quad 8.00$

$1.00 \quad 8.00$

Cystopteris bulbifera (Bladder Fern)

Cystopteris fragilis (Fragile Fern)

Dicksonia punctilobula (Hayscented Fern) strong clumps

$1.00 \quad 8.00$

Dicksonia punctilobula, Sq. Ft. sods

\begin{tabular}{l}
$1.00 \quad 8.00$ \\
\hline
\end{tabular}

$2.50 \quad 20.00$

$1.00 \quad 8.00$

$\begin{array}{rr}1.25 & 10.00 \\ 1.00 & 8.00\end{array}$

Onoclea sensibilis (Sensitive Fern)

10.00

10.00

10.00

(Cinnamon Fern)

1.25
1.25

1.25

Osmunda regalis (Royal Fern)

10.00
8.00

$\begin{array}{ll}1.00 & 8.00 \\ 1.00 & 8.00\end{array}$

Phegopteris hexagonoptera

$\begin{array}{ll}1.00 & 8.00 \\ 1.00 & 8.00\end{array}$

Phegopteris polypodioides

Polypodium vulgare (Common Polypody)

8.00

$\begin{array}{ll}1.00 & 8.00 \\ 1.00 & 8.00\end{array}$

Pteris aquilina (Common Brake)

8.00
8.00

Woodsia obtusa (Obtuse-leaved Woodsia)

12.00

Woodwardia angustifolia (Chain Fern) 


\section{HARDY PERENNIALS}

Actaea alba (White Baneberry)

Actaea rubra (Red Baneberry)

Alyssum saxatile compactum

Aquilegia canadensis

Androsace primuloides

Arabis Kelleri (a miniature rock Cress, new)

Arabis mollis

Arenaria caespitosa

Arisaema triphyllum

Asarum canadense (Wild Ginger)

Asclepias tuberosa (Butterfiy Weed)

Aster cordifolia

Aster ericoides

Aster laevis

Aster linariifolius

Aster multiflorus

Aster novi-belgii

Baptisia tinctoria

Campanula carpatica

Campanula carpatica alba

Caulophyllum thalictroides (Blue Cohosh)

Chelone glabra

Cimicifuga racemosa

Clintonia borealis

Coptis trifolia

Cornus canadensis (clumps with earth)

Dianthus deltoides

Dianthus plumarius

Dicentra canadensis

Dicentra Cucullaria

Erythronium albidum (large bulbs)

Erythronium Americanum (large bulbs)

Erythronium grandiflorum

Eupatorium ageratoides

Eupatorium perfoliatum

Eupatorium purpureum

Galax aphylla

Gaultheria procumbens (clumps with earth)

Gentiana Andrewsii

Geranium maculatum

Hepatica acutiloba (strong clumps)

Hepatica triloba

Houstonia caerulea

Hypoxis erecta

Iris cristata

Iris Pseudacorus

Iris pumila hybrida (blue)

Iris Snow Queen

Iris verna

Iris versicolor

Liatris pyenostachya

Liatris scariosa

Lilium canadense 1 st size

Lilium Grayi

Lilium philadelphicum

Lilium superbum, 1st size

Lobelia cardinalis

Lupinus perennis

Lysimachia Nummularia

Lythrum roseum superbum

Mertensia virginica, 1st size

Mitchella repens (clumps with earth)

Mitella diphylla

Myosotis palustris

Parnassia caroliniana

Phlox divaricata

Phlox reptans

Phlox subulata

Phlox subulata alba

Phlox subulata lilacina

Podophyllum peltatum

Polygala paucifolia

Polygonatum biflorum

Polygonatum majus

Primula Beesiana

Primula japonica hybrida

Sanguinaria canadensis

\begin{tabular}{|c|c|}
\hline Per 10 & Per 100 \\
\hline$\$ 1.50$ & $\$ 12.00$ \\
\hline 1.75 & 15.00 \\
\hline 1.25 & 10.00 \\
\hline 1.00 & 8.00 \\
\hline 1.25 & 10.00 \\
\hline 2.00 & 15.00 \\
\hline 1.00 & 8.00 \\
\hline 1.00 & 8.01 \\
\hline 1.00 & 8.00 \\
\hline 1.00 & 8.00 \\
\hline 1.25 & $10: 00$ \\
\hline 1.00 & 8.00 \\
\hline 1.00 & 8.00 \\
\hline 1.00 & 8.00 \\
\hline 1.00 & 8.00 \\
\hline 1.00 & 8.00 \\
\hline 1.00 & 8.00 \\
\hline 1.00 & 8.00 \\
\hline 1.00 & 8.00 \\
\hline 1.00 & 8.00 \\
\hline 1.00 & 8.00 \\
\hline 1.00 & 8.00 \\
\hline 1.00 & 8.00 \\
\hline 1.00 & 8.00 \\
\hline 1.00 & 8.00 \\
\hline 1.50 & 12.00 \\
\hline 1.00 & 8.00 \\
\hline 1.00 & 8.00 \\
\hline .75 & 6.00 \\
\hline .75 & 6.00 \\
\hline .75 & 6.00 \\
\hline .75 & 6.00 \\
\hline .75 & 6.00 \\
\hline 1.00 & 8.00 \\
\hline 1.00 & 8.00 \\
\hline 1.00 & 8.00 \\
\hline 1.25 & 10.00 \\
\hline 1.50 & 12.00 \\
\hline 1.00 & 8.00 \\
\hline 1.00 & 8.00 \\
\hline 1.25 & 10.00 \\
\hline 1.00 & 8.00 \\
\hline .75 & 6.00 \\
\hline 1.00 & 8.00 \\
\hline 1.00 & 8.0 \\
\hline 1.00 & 8.00 \\
\hline 1.25 & 10.00 \\
\hline 1.00 & 8.00 \\
\hline 1.00 & 8.0 \\
\hline 1.00 & 8.0 \\
\hline 1.00 & 8.00 \\
\hline 1.00 & 8.00 \\
\hline 1.25 & 10.00 \\
\hline 1.25 & 10.00 \\
\hline 1.25 & 10.00 \\
\hline 1.25 & 10.00 \\
\hline 1.00 & 8.00 \\
\hline 1.00 & 8.00 \\
\hline 1.00 & 8.00 \\
\hline 1.00 & 8.00 \\
\hline 1.00 & 8.00 \\
\hline 1.25 & 10.00 \\
\hline 1.00 & 8.00 \\
\hline 1.00 & $8.0 C$ \\
\hline 1.00 & $8.0 \mathrm{C}$ \\
\hline 1.00 & 8.00 \\
\hline 1.00 & \\
\hline 1.00 & 8.00 \\
\hline 1.00 & $8.0 \mathrm{C}$ \\
\hline 1.00 & 8.00 \\
\hline 1.00 & 8.00 \\
\hline 1.00 & 8.00 \\
\hline 1.00 & 8.00 \\
\hline 1.25 & 10.00 \\
\hline 1.25 & 10.00 \\
\hline 1.25 & 10.00 \\
\hline .75 & 6.00 \\
\hline
\end{tabular}


Saxifraga virginiensis

Sedum acre

Sedum album

Sedum lydium

Sedum reflexum

Sedum sexangulare

Sedum stoloniferum

Sedum stoloniferum coccineum

Sempervivum Alberti

Sempervivum arachnoideum

Sempervivum globiferum

Sempervivum tectorum

Shortia galacifolia

Silene virginica

Smilacina bifolia

Smilacina racemosa

Solidago canadensis

Solidago memoralis

Thalictrum dioicum

Thalictrum cornuti

Thymus azoricus

Thymus lanuginosus (Wolly Thyme)

Thymus serpyllum

Thymus serpyllum album

Thymus serpyllum coccineum

Tiarella cordifolia

Tradescantia virginiana

Trillium erectum

Trillium erythrocarpum

Trillium grandiflorum ( $\$ 30$ per $\mathrm{M}$ )

Trillium stylosum

Uvularia perfoliata

Vernonia noveboracensis

Veronica prostrata

Vinca minor (field grown clumps)

Viola blanda

Viola canadensis

Viola canina sylvestris

Viola cornuta

Viola cucullata

Viola Johnny-Jump-Ups

Viola lutea

Viola palmata

Viola pedata

Viola pubescens

Viola septentrionalis

\section{BOG PLANTS}

Acorus calamus

Per 10 Per 100

Calla palustris

Caltha palustris

Dionaea muscipula (Venus Fly-trap)

Menyanthes trifoliata

Sarracenia flava

Sarracenia rubra

Sarracenia purpurea

Scirpus atrovirens

Vaccinium macrocarpon

$\begin{array}{rr}\$ 1.00 & \$ 8.00 \\ .75 & 6.00 \\ .75 & 6.00 \\ 1.50 & 15.00 \\ .75 & 6.00 \\ 1.25 & 10.00 \\ 1.25 & 10.00 \\ 1.00 & 8.00 \\ .75 & 6.00 \\ .75 & 6.00\end{array}$

\section{NATIVE ORCHIDS}

Cypripedium acaule (1 to 2 crowns)

Per 10 Per 100

$\$ 1.25 \$ 10.00$

$2.50 \quad 20.00$

$5.00 \quad 40.00$

Cypripedium spectabile ( 3 to 4 crowns)

Cypripedium spectabile (5 to 8 crowns)

10.00

Goodyera pubescens

Habenaria ciliaris

$1.00 \quad 8.00$

$1.00 \quad 8.00$

Pogonia ophioglossoides

1.00

8.00

\section{EVERGREEN SHRUBS}

Prices include balling and burlapping

Andromeda floribunda 12-15 in. spread

Per 10 Per 100

Andromeda floribunda 15-18 in. spread

$\$ 17.50 \quad \$ 150.00$

(My Andromedas are heavily budded) 
Calluna vulgaris (Heather) 6-8 in.

Calluna vulgaris 8-12 in.

Calluna vulgaris $12-15$ in.

Calluna vulgaris 15-18"

10.00

Ilex glabra (Inkberry) $12-15$ in. $\quad 12.50$

Ilex glabra $15-18$ in.

17.50

$8.50 \quad 75.00$

Kalmia latifolia $12-15$ in.

$12.50 \quad 100.00$

Kalmia latifolia 15-18 in. $\quad 12.50$

Kalmia latifolia 18-24 in.

150.00

$20.00 \quad 175.00$

Kalmia latifolia $2 \mathrm{ft}$.

Leucothoe catesbaei (Drooping Leucothoe) bushy clumps 12-15 in.

$6.50 \quad 50.00$

$8.50 \quad 75.00$

$1.25 \quad 10.00$

Leucothoe catesbaei $15-18$ in.
Pachysandra terminalis 2 yr.

All Rhododendrons in nursery grown grades have been grown from stock cut back to the ground; all new tops; bushy, with fine foliage.

Rhododendron carolinianum 12-15 in.

Rhododendron carolinianum 15-18 in.

$\begin{array}{rr}\$ 12.50 & \$ 100.00 \\ 16.50 & 145.00 \\ 22.50 & 200.00 \\ 12.50 & \\ 10.00 & 85.00 \\ 12.50 & 100.00\end{array}$

Rhododendron carolinianum 18 in.

Rhododendron catawbiense $1-11 / 2 \mathrm{ft}$.

Rhododendron maximum 12-15 in.

Rhododendron maximum 15-18 in.

Rhododendron maximum 18-24 in.

16.00

140.00

Freshly collected stock at my nursery. B\&B

Rhododendron maximum $2-3$ ' B\&B

Rhododendron maximum $3-4^{\prime} \mathrm{B} \& \mathrm{~B}$

Rhododendron catawbiense 18-24" B\&B

Rhododendron catawbiense $2-3^{\prime} \quad B \& B$

15.00

20.00

15.00

Rhododendron catawbiense $3-4^{\prime}$ B\&B

20.00

125.00

175.00

125.00

These Rhododendrons are high grade material the best I have ever handled and are suitable for immediate resale to discriminating retail buyers.

\section{DECIDUOUS SHRUBS}

The prices on Azaleas include balling and burlapping

Azalea calendulacea $12-15$ in.

Per 10 Per 100

Azalea calendulacea $15-18$ in.

$\$ 10.00 \quad \$ 85.00$

Azalea calendulacea $18-24$ in.

$12.50 \quad 100.00$

Azalea canescens 12-15 in.

$16.50 \quad 150.00$

Azalea canescens 15-18 in.

$10.00 \quad 85.00$

Azalea canescens 18-24 in.

$12.50 \quad 100.00$

Azalea nudiflora $15-18$ in.

$16.50 \quad 150.00$

Azalea nudiflora 18-24 in.

10.00

15.00

$\begin{array}{rr}10.00 & 85.00 \\ 12.50 & 100.00\end{array}$

Azalea viscosa $18-24$ in.

Azalea viscosa $3 \mathrm{ft}$. selected

17.50

150.00

My Azaleas are all well budded.

Amelanchier canadensis 2-2 $\frac{1}{2}{ }^{\prime}$

Clethra alnifolia 18-24"

$\$ 2.00 \$ 15.00$

$2.00 \quad 15.00$

Diervilla trifida 18 in.

Gaylussacia resinosa 9-12"

$1.50 \quad 12.00$

Hamamelis birginiana 18-24"

$2.50 \quad 20.00$

$1.50 \quad 12.00$

Myrica gale 18 in.

12.00

$\begin{array}{ll}1.50 & 12.00\end{array}$

Rhus aromatica 18-24"

$\begin{array}{ll}2.50 & 20.00 \\ 1.50 & 12.00\end{array}$

Rhus aromatica 2-3'

Rosa blanda 15-18"

Rosa lucida 12-18 in.

$1.50 \quad 12.00$

Viburnum Lentago $3-4^{\prime}$

25.00

Viburnum dentatum 2-3'

$\begin{array}{ll}3.00 & 25.00 \\ 2.50 & 20.00\end{array}$

\section{Gillett's Ferin and Flower Farm}

KENNETH E. GILLETT, Proprietor Southwick, Massachusetts 